\title{
On those Principles of Mechanics which depend upon Processes of Variation.*)
}

By

Phitip E. B. Jourdain of Broadwindsor (England).

Chief among the questions of mathematical interest, and the points of controversy, about the variational principles of mechanics are those concerning the exact meaning of the process of 'variation' used; - whether it can be regarded as a literal (proper) variation, as considered in the calculus of variations, or not. These questions are:

(a) As to the relation of the principle of least action to Hamilton's principle, and the connected one as to whether the variable $t$ is to be varied in the former (in the latter, $\delta t=0$ );

(b) As to the extent of the principles, - whether, and under what conditions, they are applicable to the cases of non-existence of a forcefunction, non-holonomous conditions, and conditions which depend on $t$ explicitly, - and the connected difficulty as to the transformation of the principles from rectangular to general coordinates. $* *$ )

Since the time of Lagrange, both questions have arisen, in a more or less explicit form, in the work of Rodrigues, Jacobi, Ostrogradsky, Routh, A. Mayer, Sloudsky, Bertrand, Voss, Helmholtz, Réthy, Hölder, Appell, and myself, The historical part, I propose to deal with elsewhere; here I will try, with especial reference to some recent papers of Réthy***), to give what seem to me to be satisfactory answers to both questions.

*) Siebe die Arbeiten der Herren Jourdain und Réthy in den Bänden 62 und 64 dieser Annalen. Da sich auch Herr Réthy mit den neuen Ausführungen Herrn Jourdains einverstanden erklärt hat, so schliøßen wir die Diskussion.

D. Red.

**) I find it convenient to call general coordinates 'generalised' ones when they are mutually independent.

***) „Ưber das Prinzip der Aktion und über die Klasse mechanischer Prinzipien, der es angehört", Math. Ann. Bd. 58, 1904, pp. 169-194; "Bemerkungen zur Note 
1.

With regard to the questions (a), various views as to the relation of the principle of least action to Hamilton's principle have been taken by different people, or by the same person at different times. Owing to the impossibility of preserving the mutual independence of the variations of the generalised coordinates with the supposition that the quantity $T-U$ is to be unvaried in the variation when $t$ is taken as the independent variable (so that $\delta t=0$ ), Jacobi gave the principle of least action a form in which $t$ is eliminated, so that it is quite distinct from, and less general then, Hamilton's principle*). Secondly, in view of this, Lagrange's principle of least action seemed to Ostrogradsky and to Mayer in his earlier (1877) work**) to be an incorrect formulation of Hamilton's principle. Thirdly, Rodrigues, in a memoir of 1814 which had been overlooked by most until much later, had shown that the above difficulty could be surmounted, and Lagrange's principle conceived as one quite different from Hamilton's, but approaching it - and deviating from Jacobi's - in form, just as Lagrange seems to have intended, if only we vary $t$ (do not assume $\delta t=0$ ). This view was accepted by Routh and Sloudsky, and by Mayer in 1886, with explicit abandonment of his former view. Fourthly, there is Helmholtz's view that Hamilton's principle is a form of Lagrange's principle. Helmboltz is only right if suggestion of Hamilton's principle contained in the equations of condition do not depend explicitly on $t$. And lastly, there is a perfectly correct identity, established by Réthy, and showing more clearly this result of Helmholtz's.

If the equations of condition do not contain $t$ explicitly, we have, as Réthy remarked,

$$
\delta T \equiv \sum_{\nu} \frac{\partial T}{\partial q_{v}} \delta q_{\nu}+\sum_{v} \frac{\partial T}{\partial \dot{q}_{v}} \frac{d \delta q_{\nu}}{d t}-2 T \frac{d \delta t}{d t}
$$

where the $q_{\nu}$ 's are generalised coordinates. Putting

$$
\delta_{t} T \equiv \delta T+2 T \frac{d \delta t}{d t}
$$

des Herrn Philip E. B. Jourdain über das Prinzip der kleinsten Aktion", ibid., Bd. 64, 1907, pp. $156-159$.

*) In this form, 'the principle of least action' has been given in most text-books since Jacobi's time; for example, Darboux, 'Leçons sur la théorie générale des surfaces', t. 2, Paris 1889, pp. 491-500; A p pell, 'Traité de mécanique rationnelle', $2^{\circ}$ ed., t. 2., 1904, pp. 425-429; and Maggi, 'Principii della teoria matematica del movimento dei corpi, Milano, 1896, pp. 394-396.

**) „Geschichte des Prinzips der kleinsten Aktion", Akad. Antrittsvorlesung, Leipzig, 1877, p. 27-29. 
so that the variation $\delta_{t} T$ is formed as if $\delta_{t} t=0$, we see that.

$$
\delta(2 T \cdot d t) \equiv\left(\delta T+\delta_{t} T\right) d t .
$$

Réthy obtained this identity, supposing that $T$ is a homogeneous quadratic function of the $\dot{q}_{v}$ 's, and $\delta_{t}$ 'denotes a variation such that $\left.\delta t=0^{*}\right)$, and hence:

$$
\delta \int_{t_{0}}^{t_{1}} 2 T \cdot d t-\int_{t_{0}}^{t_{2}}\left(\delta T-\sum_{\nu} Q_{v} \cdot \delta q_{v}\right) d t \equiv \int_{t_{0}}^{t_{2}}\left(\delta_{t} T+\sum_{v} Q_{v} \cdot \delta q_{v}\right) d t
$$

Thus he concluded that the requirement that the variation of the actionintegral vanishes for all continuous virtual displacements $\delta q_{v}$ which vanish at the limits of integration, prenising that we have eliminated $\delta t$ by the equation

$$
\delta T-\sum_{v} Q_{v} \cdot \delta q_{v}=0
$$

is identical with Hamilton's principle:

$$
\int_{t_{0}}^{t_{1}}\left(\delta_{t} T+\sum_{v} Q_{v} \cdot \delta q_{v}\right) d t=0
$$

The identity (1) implies that the conditions do not contain $t$ explic$\left.i t l{ }^{* *}\right)$ : let us consider $T$ as a function of the $3 n$ rectangular coordinates, so that we gain generality, since $T$ is homogeneous even if the conditions contain $t$. We then have

$$
\delta \int_{t_{0}}^{t_{1}} 2 T \cdot d t-\int_{t_{0}}^{t_{1}}\left(\delta T-\delta^{\prime} U\right) d t \equiv \int_{t_{0}}^{t_{1}}\left(\delta_{t} T+\delta^{\prime} U\right) d t
$$

where the $\delta x_{r}$ 's are connected by the equations of condition. If, now, we suppose that the system has $k$ degrees of freedom, and

$$
x_{r}=\varphi_{r}\left(q_{1}, q_{2}, \cdots, q_{k}, t\right) \quad r=1,2, \cdots, 3 n,
$$

we see, by a simple calculation, that the right-hand side of (1), when equated to zero, is Hamilton's principle only if

$$
2(T) \frac{d \delta t}{d t} \equiv \sum_{v} \dot{q}_{\nu} \frac{\partial(T)}{\partial \dot{q}_{\nu}} \frac{d \delta t}{d t}-\frac{\partial(T)}{\partial t} \delta t
$$

*) Math. Ann. Bd. 58, 1904, pp. 171-172.

**) This condition is, thus, necessary (and sufficient) for the formal identity of the two principles in question. 
where $(T)$ is what $T$ becomes when all the $x$ 's are expressed in terms of the $q$ 's.*) Now (3) implies that the conditions do not depend on $t$.

The equation got by putting either side of (1) equal to zero is**) the common source of both Hamilton's principle and the principle of least action, provided that we form $\delta(T)$ by putting

$$
\delta x_{r}=\sum_{v}^{\partial \varphi_{r}} \frac{\partial q_{v}}{\partial^{2}} \delta q_{v}
$$

where the $x_{r}$ 's are determined by such equations as (2), so that $\delta x_{v}$ is a virtual displacement, in the variation

$$
\delta T \equiv \sum_{r} m_{r} \dot{x}_{r} \frac{d \delta x_{r}}{d t}-2 T \frac{d \delta t}{d t}
$$

but then $\delta(T)$ is not a 'variation' properly so called, for we do not have

$$
\delta(T)=\sum_{v}\left(\frac{\partial(T)}{\partial q_{v}} \delta q_{v}+\frac{\partial(T)}{\partial \dot{q}_{v}} \delta \dot{q}_{v}\right)+\frac{\partial(T)}{\partial t} \delta t
$$

but the expression given in (21) of $\S 3$.

Thus Helmholtz's (Lagrange's) generalised integral of least action is none other, in form, than half the integral which is fundamental in Hölder's**) work, and which includes a generalised Hamilton's principle and a generalised principle of least action, valid even where the conditions depend on $t$ explicitly. But in Hölder's method we give up, in many cases, the supposition that the $\delta$-process is a process of variation, strietly speaking. On the other hand, we shall next see how, even when the

*) Cf. $\S 3$ below.

**) See Hölder, „Über die Prinzipien von Hamilton und Maupertuig“, Gött. Nachr., 1896, pp. 122-157 and $\$ 3$ below. Only Réthy's manner of expression leads to the confusion of his view with that of Ostrogradsky; in reality, his view is that of Rodrigues and Mayer (1886), and, under these limitations and extensions as to the equations of condition, of Hölder. Hölder's general principle is

$$
\int_{t_{0}}^{t_{2}}\left(\delta T+2 T \frac{d \delta t}{\bar{d} t}+\delta^{\prime} U\right) d t=0,
$$

Hamilton's principle results from this when the $\delta$-process is further defined by the condition $\delta t=0$, and the principle of least action results when the $\delta$-process is defined, by the condition, $\delta^{\prime} U=\delta T$ ( $\delta t$ is not zero). Thus these two last principles are fundamentally quite distinct. Further, it was indicated by Hölder that the transformation into general coordinates was to be carried out in the way developed in $\S 3$ below. 
conditions depend on $t$, a literally 'variational' process can be followed. This was done by Réthy and Voss, but earlier traces are found with Routh.*)

2.

We enter, now, more fully into the class (b) of questions.

Mathematicians have had to face the difficulties introduced into the formulation of the variational principles of mechanics by two generalising suppositions: that the equations of condition contain $t$ explicitly; and that some of the equations of condition are not integrable (the mechanical system is not holonomous).*) Two methods have been proposed. The one, contained in germ in the works of Routh, and developed, apparently independently of Routh, by Réthy and, independently of Réthy but not of Routh, by Voss (1900), preserves the strictly variational character of the $\delta$ used even when the conditions contain t; the other, due to Hölder and followed by $\mathrm{me}$, in the formulation of the principles for general coordinates ***), abandons the strictly variational character of the $\delta$ used $\dagger$ ) -, although Hölder's 'variations' are closely connected with variations in the strict sense -, and then deduces, by somewhat simpler considerations $\dagger^{\dagger}$ ), a general 'variational' principle, including both a generalised Hamilton's principle and a generalised principle of least action, valid when both of our above generalising suppositions are made.

*) In his 'Rigid Dynamics' since 1877; cf. 6 th ed. (1905) of Part II ('Advanced Part'), pp. 301 sqq.

**) Other generalising suppositions are: (1) The force-function depends explicitly on $t$, and also may on the $\dot{q}_{\nu}$ 's; (2) no force-function exists, but the $Q_{\nu}$ 's depend on the $q_{v}$ 's alone; (3) the same, with the $Q_{v}$ 's depending on the $q_{\nu}$ 's and $t$, and possibly the $\dot{q}_{v}, ' s ;(4)$ the differential equations of condition are not linear (for example, if a point is constrained to move on the surface of a cone, the differential equation of condition is not linear when the point is at the apex); (5) the conditions are expressed by inequalities. Here we do not consider (1), (3), (4), or (5); but (2) is introduced here. Of course, strictly speaking, we can only have a variation $(\delta U)$ if a force-function $U$ exists.

***) Voss had intended to do this in his paper of 1900 but, without realising it, substituted Routh's (completely different) point of view of obtaining virtual displacement.

t) Hölder was quite conscious of this fact (see a note in Quart. Journ. of Math., 1904, p. 75).

+f) I mean the deduction of a virtual displacement from the formula

in the form

$$
\delta x=\sum_{v} \frac{\partial x}{\partial q_{v}} \delta q_{v}+\frac{\partial x}{\partial t} \delta t
$$

$$
\delta x-\frac{\partial x}{\partial t} \delta t .
$$


The abandonment of the strict conception of a 'variation' may appear to be a disadvantage. But it seems to me that this is compensated by greater simplicity; while, in any case, when we come to deal with nonholonomous systems, we must abandon this strict conception, as was pointed out by Voss in 1884 and by others later, in somewhat differont forms. *) Lastly, unless the conditions do not contain $t$, the form of Réthy and Voss requires a condition **) holding for $\delta t$ at the limits $\left(t=t_{0}, t_{1}\right)$, whereas, in Hölder's generalised principle of least action, no such condition is required.

These advantages appeared to me - and still appear to me - to be decisive in choosing Hölder's process of 'variation' in these generalised principles in preference to the other***). But, when I stated $\dagger$ ) that $\delta x-\frac{\partial x}{\partial t} \delta t$ is a virtual displacement and that $\delta x-\dot{x} \cdot \delta t$, the displacement used by Routh, Réthy, and Voss, is not, I had not realised that two funda-

*) C. Neumann (1888), Hertz (1894), Hölder (1896) und Appell (1898); see also Boltzmann, „Vorlesungen über die Prinzipe der Mechanik“", Teil II, Leipzig 1904, pp. 30-34.

**) Namely, that

$$
\left.\left(2 T-\sum_{v} \dot{q}_{v} \frac{\partial T}{\partial \dot{q}_{v}}\right) \delta t\right|_{t_{0}} ^{t_{1}}=0 .
$$

If the equations do not depend explicitly on $t$,

$$
\sum_{v} \dot{q}_{v} \frac{\partial T}{\partial \dot{q}_{v}}=2 T
$$

and the above condition for $\delta t$ at the limits of integration is unnecessary.

***) Hölder's 'variation' becomes a variation in the strict sence when: (1) Hamilton's principle is applied, and the conditions are holonomous; or (2) the principle of least action is applied, and the conditions are holonomous, and independent of $t$. With Réthy, as has been remarked, the variation is a literal one even when the last restriction in (2) is taken away.

†) Quart. Journ. of Math., 1904, pp. 72, 75; Math. Ann. Bd. 62, 1906, pp. 415, $417-418$.

Réthy's remark (Math. Ann. Bd. 64, 1907, pp, 156-157) that my statement that $\delta \int_{t_{0}}^{t_{1}} 2 T \cdot d t=0$ is only true, without further discussion, if the conditions do not contain $t$ explicitly, is correct if $\delta$ is a literal variation; not correct if (as I assumed) $\delta$ is a Hölder's 'variation'. It would have been better not to write $\delta \int_{t_{0}}^{t_{2}} 2 T \cdot d t=0$, but to keep the form: $\int_{t_{0}}^{t_{2}}\left(2 T \cdot d \delta t+2 \delta_{1} T \cdot d t\right)=0$, where $\delta_{1} T$ is a 'variation' (not strictly speaking) defined by Hölder's process; but I followed Hölder's precedent, and also that in Encykl. der math. Wiss. IV 1, p. 93.

My investigations (Quart. Journ. of Math., 1905, pp. 290-294) also use Hölder's $\delta$-process, exclusively. 
mentally different kinds of 'variation' were used. That this is so, I trust I have made quite clear in the present paper: it seems that this fact has hitherto been overlooked, or only perceived obscurely. For my own part, this only became quite clear to me in consequence of two letters which Prof. Hölder wrote to $\mathrm{e}^{*}$ ), in which he communicated the results of an examination of the matter, which he had kindly undertaken with a view to clearing up the dispute between Réthy and myself. I desire here to record my grateful thanks to Prof. Hölder. My contribution to Hölder's method is the remark ( $\$ 3$ ) that the Hölder's variation of $T$ in the form I gave can be expressed in the same form when $T$ is replaced by $(T)$, the same function when reduced by means of the finite conditions (which may depend on $t$ explicitly.) This remark was necessary and sufficient for the formulation of 'Hölder's general principle in general coordinates**), for it showed that, in this respect, Hölder's generalised 'variations' behaved like ordinary variations. ***)

I now proceed to a discussion of those virtual displacements used by Routh, Voss, and Réthy, as compared with those used by Hölder and myself.

When writing down $\dagger$ ) the result of varying his fundamental integral when rectangular coordinates, between which equations of condition subsist, are used, Routh made the important remark $\dagger \dagger$ ), which we shall find used by Voss, that $\delta x-\dot{x} \delta t$, - the projection on the $x$-axis of the displacement of the particle $m$ from its position in the actual motion at the time $t$ to its position in the neighbouring motion at the same time, is a virtual displacement. In fact, suppose that, by means of the equations of condition, each $x$ can be expressed as a function of $k$ mutually independent parameters $q_{1}, q_{2}, \cdots, q_{k}$ and $t$ :

*) On Aug. 16 th and Sept. 1 st and 3rd, 1907.

**) Analogously, Voss and Réthy remarked that, where $\delta^{\prime}$ is the operator $\delta-\frac{d}{d t} \cdot \delta t$, $\delta$ being a literal variation, $\delta^{\prime}$ is a literal variation, so that it acts on both $T$ and the above $(T)$ in the same way.

***) In this paper, I always use dots to denote total differential quotients with respects $t$, and $\delta^{\prime} U$ is used as an abbreviation merely of $\sum_{r} X_{r} \cdot \delta x_{r}$, without any
implication that a force-function $U$ exists.

†) Op. cit., art. $446, \mathrm{p}, 303$.

††) Ibid. I controverted this remark in Quart. Journ. of Math., 1904, p. 75, and Math. Ann. Bd. 62, 1906, pp. 417-418, because I was under the impression that, with Routh, $\delta x$ is defined by $\sum_{\nu} \frac{\partial x}{\partial q_{v}} \delta q_{\nu}+\frac{\partial x}{\partial t} \delta t$, the $q_{\nu}$ 's being generalised coordinates; $\delta x-\frac{\partial x}{\partial t} \delta t$ is then the only expression for a virtual displacement. But see the above text, and introduction. 


$$
\left.x_{r}=\varphi_{r}\left(q_{1}, q_{2}, \cdots, q_{k}, t\right) \quad r=1,2, \cdots, 3 n^{*}\right),
$$

then, where $x$ is varied by varying the $q$ 's and $t$,

$$
\delta x_{r}=\sum_{\nu} \frac{\partial \varphi_{r}}{\partial q_{\nu}} \delta q_{\nu}+\frac{\partial \varphi_{r}}{\partial t} \delta t
$$

while

$$
\dot{x}_{r}=\sum_{v} \frac{\partial \varphi_{r}}{\partial q_{v}} \dot{q}_{v}+\frac{\partial \varphi_{r}}{\partial t}
$$

A virtual displacement $\left(\delta_{1} x\right)$ of $x$ is one which is given by

$$
\sum_{v} \frac{\partial \varphi}{\partial q_{v}} \delta q_{v}^{(1)}
$$

where the $\delta^{(i)} q^{\prime}$ 's are any variations of the $q$ 's. One such is given by

$$
\delta x-\frac{\partial \varphi}{\partial t} \delta t
$$

but another can be found by choosing, instead of the $\delta q$ 's used in some definite set (of $3 n$ equations) of the equations (5), other variations of the $q$ 's. For, noticing that, by (5) and (6),

$$
\delta x-\dot{x} \cdot \delta t=\sum_{v} \frac{\partial \varphi}{\partial q_{v}}\left(\delta q_{v}-\dot{q}_{v} \cdot \delta t\right),
$$

the terms of which $\frac{\partial \varphi}{\partial t}$ is a factor vanishing, we see that $\delta x-\dot{x} \delta t$ is virtual. The variation $\delta x$ is then defined as follows: Consider any definite set of variations $\delta^{\prime} q_{1}, \delta^{\prime} q_{2}, \cdots, \delta^{\prime} q_{k}, \delta^{\prime} t$; form the new set $\delta^{\prime} q_{1}-\dot{q}_{1} \cdot \delta^{\prime} t, \cdots, \delta^{\prime} q_{k}-\dot{q}_{k} \cdot \delta^{\prime} t$, - which are eo ipso 'virtual'**); then

$$
\sum_{\nu} \frac{\partial \varphi}{\partial q_{\nu}}\left(\delta^{\prime} q_{\nu}-\dot{q}_{\nu} \cdot \delta^{\prime} t\right)
$$

represents a virtual displacement of $x$ which we will call $\delta_{1}^{\prime} x$. Then $\delta^{\prime} x$ is defined as $\delta_{1}^{\prime} x+\dot{x} \delta^{\prime} t$; it is literally a 'variation' (which $\delta x-\frac{\partial x}{\partial t} \delta t$ is not). All the particular variations $\delta^{\prime}$ form a class; in propositions about any member (a 'variable' member, as we say), of this class', the accent may evidently be dropped.

*) For the extension to the case where some of the conditions are differential, see below.

**) By this, I mean that, since the displacement of any coordinate is 'virtual' if it satisfies the equations of condition at the instant, any displacement of the generalised coordinates is consistent with the equations of condition subsisting between them, - for there are no such equations of condition. 
We see, then, that when the conditions are holonomous, given in the form (4), the process of variation used in Hamilton's principle is, in every case, a literal variation; in the principle of least action, on the other hand, the variation is only a literal one if the equations of condition are explicitly independent of $t$.

Now, Réthy, by considerations closely connected with those of Routh, made the important remark that the principle of least action can be so formulated that it is a problem of literal variations even when the conditions contain $t$ explicitly.*)

Réthy also used a literal variation; his fundamental identity is $* *$ ), where $\Phi$ is a function of the $q$ 's, $\dot{q}$ 's, and $t$,

$$
\delta(\Phi \cdot d t)-d\left(\Phi \cdot \delta t+\sum_{v} \frac{\partial \Phi}{\partial \dot{q}_{v}} \delta^{\prime} q_{v}\right) \equiv \sum_{\nu}\left(\frac{\partial \Phi}{\partial q_{v}}-\frac{d}{d t} \frac{\partial \Phi}{\partial \dot{q}_{v}}\right) \delta^{\prime} q_{v} \cdot d t
$$

where here

$$
\delta^{\prime} q_{v} \equiv \delta q_{v}-\dot{q} \cdot \delta t
$$

If we add to the identity (7) the identity

$$
\delta(\Phi \cdot d t)-d(\Phi \cdot \delta t) \equiv \delta^{\prime} \Phi \cdot d t
$$

where $\delta^{\prime} \Phi$ is formed in analogy with (8), put $\Phi=T$, and integrate the result between $t_{0}$ and $t_{1}$, we get the (Voss') theorem together with Réthy's completion of it***):

$$
\delta \int_{t_{0}}^{t_{2}} 2 T \cdot d t \equiv \int_{t_{0}}^{t_{0}} \sum_{\nu}\left(Q_{\nu}+\frac{\partial T}{\partial q_{v}}-\frac{d}{d t} \frac{\partial T}{\partial \dot{q}_{v}}\right) \delta^{\prime} q_{v} \cdot d t
$$

*) This aspect of Réthy's work previously escaped me. I tacitly used Hölder's process of 'variation', while Réthy (strictly speaking, correctly) did not admit that it was a 'variation' at all (on this point, of which Holder was perfectly conscious, see Quart. Journ. of Math, 1904, p. 75, note). Thus I wrongly attributed to Rethy, Routh and Voss certain errors. In fact, I assumed, and still assume, the greater importance of Hölder's method of forming a concept of a (not literal) variation which remains valid for non-holonomous systems; no literally variational process being capable of this.

**) Math. Ann. Bd. 58, 1904, p. 173. I only know of his earlier (1895-1896) work from this paper.

A somewhat more general formulation, brought about by adding $c$ times the dentity $\delta(\Phi \cdot d t)-d(\Phi \cdot \delta t) \equiv \delta^{\prime} \Phi \cdot d t, c$ being an arbitrary constant, to (7) was given by Réthy in ibid., Bd. 64, 1907, pp. 157-158.

***) Voss' theorem cannot be inverted (see Math. Ann. Bd. 58, 1904, pp. 174-175), and the above invertible theorem was given in ibid., pp. 175-176 and Bd. 64, 1907, pp. $157-158$. 
if $\delta t_{0}$ and $\delta t_{1}$ are determined according to the equation

$$
\left|2 T \cdot \delta t+\sum_{v} \frac{\partial T}{\partial \dot{q}_{v}} \delta^{\prime} q_{v}\right|_{t_{0}}^{t_{1}}+\int_{t_{0}}^{t_{2}}\left(\delta^{\prime} T-\sum_{v} Q_{v} \cdot \delta^{\prime} q_{v}\right) d t=0 .
$$

If this latter equation is satisfied for any time $t_{1}$, and if the ratios $\frac{\delta q_{v}}{\delta t}$ are subject to the equations of condition subsisting between the $\dot{q}_{v}$ 's, then

$$
\delta \int_{t_{0}}^{t_{2}} T \cdot d t
$$

vanishes when, and only when, the motion is the natural one.

Voss*) proposed to apply Hölder's process of thought to the case of quite general coordinates, which Hölder**) had only briefly indicated. In view of this explicit declaration, it is surprising to see Hölder's concept of 'variation'***) nowhere used. However, Voss' method, though it is not described by him with all the clearness possible, can be understood as a solution of the problem of finding expressions such that variations (in the literal sense) of them give the equations of motion. And this had been done, in a similar way, by Réthy.

Suppose that the equations of condition are partly in the form of linear differential equations. Thus, the finite equations (which may depend explicitly on the time) being satisfied by $k$ parameters $q_{v}$, there also subsist $l$ differential equations of the form

$$
\sum_{\nu=1}^{k} p_{\mu, \nu} d q_{v}+p_{\mu} d t=0, \quad, \quad=1,2, \cdots, l
$$

where the $p$ 's are functions of the $q$ 's and $t$.

Considering $T$ as a function of these $n q_{\nu}$ 's and $t$, Voss obtained $\dagger$ ) where $\delta$ is a literal variation,

*) "Ưber die Principe von Hamilton und Maupertuis" [July, 1900], Gött. Nachr., Math.-Phys. Klasse, 1900, pp. 322-327. We need not here consider the special case first ( $\$ 1$ ) considered by Voss, that the conditions do not depend on $t$ explicitly; but will at once proceed to the general case.

**) Loc. cit., p. 14, note.

***) The $\delta x-\frac{\partial x}{\partial t} \delta t$ of $\S \S 2$ and 6 .

$\dagger$ ) Correcting a few slips in his calculation. 


$$
\begin{aligned}
\delta \int_{t_{0}}^{t_{1}} 2 T \cdot d t & \equiv \int_{t_{0}}^{t_{1}}(2 \delta T \cdot d t+2 T \cdot d \delta t) \equiv\left|\sum_{v} \frac{\partial T}{\partial \dot{q}_{v}} \delta q_{v}+\left(2 T-\sum_{v} \dot{q}_{v} \frac{\partial T}{\partial \dot{q}_{v}}\right) \delta t\right|_{t_{o}}^{t_{2}} \\
& +\int_{t_{0}}^{t_{1}} d t\left\{\delta T-\dot{T} \cdot \dot{\delta} t+\sum_{v}\left(\frac{\partial T}{\partial q_{v}}-\frac{d}{d \bar{t}} \frac{\partial T}{\partial \dot{q}_{v}}\right)\left(\delta q_{v}-\dot{q}_{v} \cdot \delta t\right)\right\}
\end{aligned}
$$

where the $\delta q_{\nu}$ 's are subject to the equations

$$
\sum_{\nu=1}^{k} p_{\mu, \nu} \delta q_{\nu}+p_{\mu} \delta t=0 \quad \mu=1,2, \cdots, l .
$$

It is evident that Hölder's line of thought is quite abandoned, and that of Routh and Réthy adopted. It was necessary, to make the above identity serve in a mechanical theorem, to so arrange that $\delta q_{y}-\dot{q}_{\nu} \cdot \delta t$ should correspond to a virtual displacement.

A virtual displacement is any system of displacements $\delta_{1} q_{1}, \delta_{1} q_{2}, \cdot$ $\cdot ., \delta_{1} q_{k}$ such that

$$
\sum_{\nu=1}^{k} p_{\mu, \nu} \delta_{1} q_{\nu}=0 \quad \mu=1,2, \cdots, l .
$$

By combining*) (9), multiplied by $\delta t$, and (10), we get

$$
\sum_{\nu=1}^{k} p_{\mu, v}\left(\delta_{1} q_{\nu}+\dot{q}_{v} \cdot \delta t\right)+p_{\mu} \delta t=0
$$

if, then, we define a new - not a virtual $* *$ ) - displacement by the relations

$$
\delta q_{\nu}=\delta_{1} q_{\nu}+\dot{q}_{\nu} \cdot \delta t
$$

*) The following elucidations of Voss' not very clearly expressed method are taken from lotters of Prof. Holder's to me (dated 16. VIII. 1907 and 1. IX. 1907).

**) If all the $n q_{v}$ 's were mutually independent, then any system of variations given to them would result in a virtual displacement of the mechanical system and any real variation of a $q_{y}$ is eo ipso virtual, as remarked in the note on $p .417$ of the Math. Ann. Bd. 62. Réthy wrongly attributed (ibid., Bd. 64, p. 158) to me the remark that $\delta q_{\nu}-\dot{q}_{v} \cdot \delta t$ is not, in general, virtual: what I said (ibid., Bd. 62, pp. 418-419) was that, if $\delta x$ is any variation (affecting $t$ also, and defined by

$$
\delta x=\sum_{v}\left(\frac{\partial x}{\partial q_{v}} \delta q_{v}+\frac{\partial x}{\partial t} \delta t\right)
$$

of $x, \delta x-\dot{x} \cdot \delta t$ is not virtual, - for $\delta x-\frac{\partial x}{\partial t} \delta t$ is. The question as to whether a certain displacement of a coordinate is 'virtual' or not can only arise when the coordinate is not independent.

Here the general case is considered: the $q$ 's fix the position of the system at the time $t$ - and, indeed, over-determine it, for further equations of condition, which need not be integrable, hold between the $d q$ 's. 
so that

$$
\delta_{1} q_{v}=\delta q_{v}-\dot{q}_{v} \cdot \delta t,
$$

then the $\delta q$ 's and $\delta t$ satisfy the same equations that the $d q$ 's and $d t$ do in the actual motion; that is to say, we have

$$
\sum_{\nu=1}^{k} p_{\mu, v} \cdot \delta q_{\nu}+p_{\mu} \cdot \delta t=0 \quad \mu=1,2, \cdots, l .
$$

If some of the relations (9) are integrable, that is to say, can be put in the form

$$
d \varphi_{\mu}\left(q_{1}, q_{2}, \cdots, q_{k}, t\right)=0,
$$

then these relations can be shown to subsist also for

$$
q_{1}+\delta q_{1}, q_{2}+\delta q_{2}, \cdots, q_{k}+\delta q_{k}, \quad t+\delta t,
$$

so that equations of condition given in a finite (integrable) form subsist also for a motion varied in the manner represented by (11). For a holonomous system, then, the motion varied in this way is a possible one, even when the conditions depend explicitly on $t$ and $\delta t$ is not zero.

Thus, Voss imagines, instead of the varied motion brought about by virtual displacements (considered by Hölder and myself), another fictitious motion, represented by (11), and, concludes that, for such a varied path, the quantity $\delta T$ can be found by direct variation of the expression $T$, and that this is also correct when the expression for $T$ is simplified by the finite equations of condition.

Hence, we get Voss' theorem*):

If $\delta^{\prime}()$ denotes the operator $\delta()-\frac{d()}{d t} \delta t$; then, if the equations

$$
\begin{gathered}
\delta^{\prime} T=\sum_{v=1}^{n} Q_{v} \cdot \delta^{\prime} q_{v}, \\
\left|2 T \cdot \delta t+\sum_{v=1}^{n} \frac{\partial T}{\partial \dot{q}_{v}} \delta^{v} q_{x}\right|_{t_{0}}^{t_{1}}=0,
\end{gathered}
$$

are considered to be conditions for the virtual displacement $\delta^{\prime} q_{\nu}$ and for the $\delta t^{\prime}$ s at the times $t_{0}$ and $t_{1}$, then

$$
\delta \int_{t_{0}}^{t_{1}} 2 T \cdot \delta t
$$

*) First stated exactly by Réthy. 
becomes the integral

$$
\int_{t_{0}}^{t_{2}} d t \sum_{\nu=1}^{n}\left(Q_{\nu}+\frac{\partial T}{\partial q_{v}}-\frac{d}{d t} \frac{\partial T}{\partial q_{v}} \cdot\right) \delta^{\prime} q_{\nu},
$$

where the $\delta^{\prime} q_{\nu}^{\prime}$ 's are subject to the equations (10) and (12).

\section{3.}

I now come to my own derivation of the equations of motion in quite general coordinates from Hölder's general principle.

Suppose that there are $l$ equations between the $3 n x_{r}$ 's, and $t$ of the form

$$
\Psi_{i}\left(x_{1}, x_{2}, \cdots, x_{3 n}, t\right)=0 \quad i=1,2, \cdots, l,
$$

and suppose that these finite equations are all identically satisfied by $k$ parameters $q_{1}, q_{2}, \cdots, q_{k}$, where $k=3 n-l$. Thus we have $3 n$ equations:

$$
x_{r}=\varphi_{r}\left(q_{1}, q_{2}, \cdots, q_{k}, t\right) \quad r=1,2, \cdots, 3 n .
$$

Let us further suppose that there are, besides (13), $l^{\prime}$ non-integrable relations between the $d x^{\prime}$ s and $d t$, or, what comes to the same thing, $l^{\prime}$ relations

$$
\sum_{\nu=1}^{k} \xi_{\nu}^{(i)} \cdot d q_{\nu}+\xi^{(i)} \cdot d t=0 \quad i=1,2, \cdots l^{\prime}
$$

between the $d q$ 's and $d t$.

The variation, $\delta T$, of $T$ is

$$
\sum_{r=1}^{3 n} m_{r} \dot{x}_{r} \frac{d \delta x_{r}}{d t}-2 T \frac{d \delta t}{d t}
$$

and, in the principle of Hölder:

$$
\int_{t_{0}}^{t_{2}}\left(\delta T-2 T \cdot \frac{d \delta t}{d t}+\delta^{\prime} U\right) d t=0
$$

these displacements $\delta x_{r}$ are restricted to represent virtual displacements. If we use the equations (14) to eliminate the $\dot{x}_{r}$ 's from $T, T$ becomes, we will say, $(T)$, a function, in general, of the $q$ 's, $\dot{q}$ 's, and $\left.t^{*}\right)$, and whereas, then, a variation of $x_{r}$ :

*) This distinction of $T$ and $(T)$ is useful, for we have:

$$
\frac{\partial T}{\partial x_{r}}=\frac{\partial T}{\partial t}=0, \quad \sum_{r=1}^{3 n} \dot{x}_{r} \frac{\partial T}{\partial x_{r}}=2 T
$$




$$
\delta x_{r}=\sum_{v=1}^{k} \frac{\partial \varphi_{r}}{\partial q_{v}} \delta q_{v}+\frac{\partial \varphi_{v}}{\partial t} \delta t
$$

is not, in general, a virtual displacement, since a displacement $\left(\delta_{1} x_{r}\right)$ is only virtual if displacements $\delta_{1} q_{1}, \delta_{1} q_{2}, \cdots, \delta_{1} q_{k}$ exist such that

$$
\delta_{1} x_{r}=\sum \frac{\partial \varphi}{\partial q_{v}} \delta_{1} q_{r}
$$

where

$$
\sum_{\nu=1}^{k} \xi_{v}^{(i)} \delta_{1} q_{\nu}=0 \quad i=1,2, \cdots, l^{\prime}
$$

For a definite set of the variations $\delta q_{v}, \delta t$ used in (16), the displacement

$$
\delta x_{r}-\frac{\partial \varphi_{r}}{c t} \delta t
$$

which, however, is not a 'variation' in the strict sense of the word, is virtual with respect to the finite equations (14), that is to say, if we disregard, for the moment, the differential conditions (15) and the conditions (18) that the displacement of the $q_{v}$ 's must satisfy when (15) are considered. In this case the $\delta T$ in Hölder's principle, does not become a rariation of $(T)$, such that

$$
\delta(T)=\sum_{v}\left(\frac{\partial(T)}{\partial q_{v}} \delta q_{v}+\frac{\partial(T)}{\partial \dot{q}_{v}} \delta \dot{q}_{v}\right)+\frac{\partial(T)}{\partial t} \delta t
$$

but what

$$
\delta T \equiv \sum_{r} m_{r} \dot{x}_{r} \frac{d \delta x_{r}}{\partial t}-2 T \frac{d \delta t}{d t}
$$

becomes when for the $\delta x_{r}$ 's are put the virtual displacements given by (19), so that the $\delta T$ in Hölder's principle becomes, in terms of $(T)$,

whereas we do not, in general, have:

$$
\frac{\partial(T)}{\partial q_{\nu}}=0, \frac{\partial(T)}{\partial t}=0, \text { or } \sum_{\nu=1}^{k} \dot{q}_{\nu} \frac{\partial(T)}{\partial \dot{q}_{\nu}}=2(T) .
$$

However, when the conditions do not contain $t$, we have

$$
\frac{\partial(T)}{\partial t}=0, \quad \sum_{\nu=1}^{t} \dot{q}_{\nu} \frac{\partial(T)}{\partial \dot{q}_{\nu}}=2(T) ;
$$

though the second equality is not a consequence of the first. 


$$
\begin{aligned}
& \sum_{r} m_{r} \dot{x}_{r} \frac{d}{d t}\left(\sum_{\nu}^{\frac{\partial x_{r}}{\partial q_{v}} \delta q_{v}}\right)-2(T) \frac{d \delta t}{d t} \\
\equiv & \left.\sum_{v}\left(\frac{\partial(T)}{\partial q_{v}} \delta q_{v}+\frac{\partial(T)}{\partial \dot{q}_{v}} \frac{d \delta q_{v}}{d t}\right)-2(T) \frac{d \delta t}{d t} *\right),
\end{aligned}
$$

which, when transformed into

$$
\sum_{v}\left(\frac{\partial(T)}{\partial q_{v}} \delta q_{v}+\frac{\partial(T)}{o} \dot{q}_{v} \delta \dot{q}_{v}\right)+\left(\sum_{v} \dot{q}_{v} \frac{\partial(T)}{\partial \dot{q}_{v}}-2(T)\right) \frac{d \delta t}{d t}
$$

shows, by comparison with (20), that this expression (21) is not a literal variation of $(T)$. What I remarked**) was that the $\delta T^{* * * *}$ ) in Hölder's principle (19) can be expressed in the form (21), which, as we shall see, makes the introduction of general coordinates easy, even though we have not to deal with variations properly so called, when $T$ is reduced to $(T)$ by the finite equations of condition, which, in general, contain $t$ explicitly.

Since, then also, the $\delta^{\prime} U$ in Hölder's general principle transforms into $\sum_{v} Q_{v} \cdot \delta q_{v}$ by (16) and (19), and introducing now the differential conditions, Hölder's principle, where the $\delta x^{\prime}$ s are subject to the conditions (17) and (18), transforms into the principle

$$
\int_{i_{0}}^{t_{2}} d t \sum_{v}\left(Q_{v}+\frac{\partial T}{\partial q_{v}}-\frac{d}{d t} \frac{\partial T}{\partial \dot{q}_{v}}\right) \delta q_{v}=0
$$

where the $\delta q_{v}^{\prime}$ 's are subject to the conditions

$$
\sum_{r} \xi_{\nu}^{(i)} \cdot \delta q_{r}=0 \quad i=1,2, \cdots, l^{\prime}
$$

From these equations we get, in the well-known way, the equations of motion to be

$$
Q_{\nu}+\frac{\partial(T)}{\partial q_{v}}-\frac{d}{d t} \frac{\partial(T)}{\partial \dot{q}_{v}}+\sum_{i=1}^{z^{\prime}} \lambda \cdot \zeta^{(i)}=0 \quad \nu=1,2, \cdots, k,
$$

where the $\lambda$ 's are Lagrange's multipliers.

The Manor House, Broadwindsor, Dorset. Dec. 30 th 1907 and March $10^{\text {th }}, 1908$.

*) In this deduction, we make use of the finite (non-differential) character of the equations (14) by putting

$$
\frac{\partial \dot{x}}{\partial \dot{q}}=\frac{\partial x}{\partial q}, \quad \frac{\partial \dot{x}}{\partial q}=\frac{d}{d t} \frac{\partial x}{\partial q} .
$$

It is for the reason that we use these equations that we consider first exclusively the holonomous part of the conditions.

*) Prof. Hölder tells me that he had not remarked this (Sept. 3rd, 1907).

* Denoted $\delta_{1} T$ by me in Math. Ann. Bd. 62, 1906, p. 416. 